\title{
Percepção e utilização dos conteúdos de saúde coletiva por cirurgiões-dentistas egressos da Universidade Federal de Goiás
}

\author{
Perceptions and use of collective health \\ by surgeon dentists egress from Federal University of Goiás
}

Denise Elisabeth de Campos Badan ${ }^{1}$

Vânia Cristina M arcelo ${ }^{1}$

Dais Gonçalves Rocha ${ }^{1}$

${ }^{1}$ FaculdadedeO dontologia, UniversidadeFederal de Goiás. Av. Universitária Esquina com 1aㅡenida $\mathrm{s} / \mathrm{n}$, Setor Universitário. 74605-220 Goiânia GO. debadan@gmail.com
Abstract The present time is demanding for the dentists the challenge of reviewing the conception of health attention. The Brazilian National Curriculum Guidance (DCN) suggests changes in graduation, strengthening SUS. The aim of this study was to know the perceptions and uses of the collective health knowledge in the daily practice of the dentists graduated at the Federal U niversity of Goiás in the years of 2000, 2001 and 2002. It was used the methodology of triangulation overlapping technique. Results indicated that $83.3 \%$ of the graduated are working as dentists. After graduation, $68.1 \%$ attended post graduation courses, specialization. They have doubts about collective health actionsin spite of saying that practice them. This indicates that the terminology of collective health still remain unclear. Those who work in the public health system accomplished more actions in the field. They related, as difficulties, the lack of complementary material and little valorization by the population of this kind of activity. The most remembered item was health promotion $(100 \%)$ and the most used in the practice were education and prevention (60\%). The practices during the practical stages were considered very important. It is necessary to be clearer during the course about the collective health practices and deeper curriculum integration.

Key words Publichealth, Dental education, Dentistry curriculum, Public health dentistry
Resumo A atualidade tem exigindo dos profissionais cirurgiões-dentistas o desafio de rever 0 conceito de atenção à saúde. As Diretrizes Curriculares $\mathrm{N}$ acionais (DCN) sugerem mudanças na graduação, incentivando a consolidação do SUS. Objetivou-se conhecer a percepção e utilização dos conteúdos de saúde coletiva na prática dos egressos de 2000 a 2002, do curso de odontologia da U niversidade Federal de Goiás. U tilizou-se a metodologia da triangulação de técnicas. Encontrou-se que $83,3 \%$ dos egressos trabalham como cirurgião-dentista. Continuaram os estudos cursando pós-graduações e especializações $(68,1 \%)$. Têm dúvidas sobre as ações em saúde coletiva, embora as pratiquem. Atuar no serviço público determinou realizações demais práticas em saúde coletiva. Os principais entraves ao desenvolvimento de ações em saúde coletiva foram falta de recursos materiais complementares e dificuldade de valorização pela população. 0 conteúdo recordado principal foi a promoção da saúde(100\%) e o mais utilizado foi a prevenção, seguida de educação em saúde. As práticas de estágio extramuros foram muito valorizadas. Conclui-se que há necessidade de maior clareza, durante a graduação, sobre o que sejam práticas em saúde coletiva e maior integração curricular na graduação em odontologia.

Palavras-chave Saúde coletiva, Educação em O dontologia, Currículo de odontologia, Odontologia em saúde pública 
Introdução

O mundo contemporâneo, dinâmico, exige que seja revisto, como um desafio, o paradigma de se oferecer saúde não apenas tratando as doenças. É preciso que haja uma reformulação na definição de necessidades, que rompa a restrita visão técnica e individualista das causas de saúde e doença. O profissional de saúde contemporâneo deverá pensar eatuar em seu meio de forma mais crítica e reflexiva considerando o contexto onde estão inseridos profissional e paciente. Ao tratar dos serviços de saúde, Sheiham et al. ${ }^{1}$ sintetizam que deve haver um mínimo de necessidade de saúde bucal não atendida e que um máximo de saúde bucal seja alcançado por todos. Freire ${ }^{2}$ complementa: É necessário atuar a nível multissetorial, propondo açõesquediminuam a desigualdade social, melhorem o nível deinformação, estimulem práticas saudáveis com recursos locais e promovam melhorias, tanto na saúde bucal como na saúde ge ral da população. Cada vez mais, o cirurgião-dentista depara-se com a oportunidadee necessidade de atuar em áreas que ultrapassam as barreiras do consultório odontológico tradicional ${ }^{3}$. Uma dessas áreas tem sido denominada de saúde coletiva. A expressão saúde coletiva não nasceu pronta, ao contrário, é um desafio ainda em construção, fruto de muita reflexão ao longo dos anos. No caso específico daAmérica Latina, a emergência nos últimos vinte anos de um campo que se designou como saúde coletiva permite a identificação de pontos de encontro com os movimentos de renovação da saúde pública institucionalizada, seja como campo científico, seja como âmbito de práticas e mesmo como atividade profissional ${ }^{4}$. A tual mente, os aspectos norteadores das ações em saúde coletiva indicam ações para além dos limites biológicos do indivíduo, consideram também o contexto social. $E$, dessa forma, o conhecimento vai sedelineando não apenas pelo puro contato com a realidade, mas na medida do reconhecimento, compreensão e contextualização de aspectos dessa realidade, conjugados com iniciativas conducentes ao desejo de transformação.

Assume-se, nesse trabalho, a visão de saúde bucal coletiva como sendo um campo de conhecimentosepráticas integrando um conjunto mais amplo identificado como 'saúde coletiva' e que, a um só tempo, compreende também o campo da 'odontologia', incorporando-o e redefinindo-o e, por esta razão, necessariamente transcendo- $0^{5}$.

0 artigo 4 - da Resolução $n$ ㅇ CNE/CES 03, que institui Diretrizes Curriculares $\mathrm{N}$ acionais do curso de graduação em odontologia (DCNO), ex- plicita que a formação do cirurgião-dentista objetiva dotar o profissional de conhecimentos, competências e habilidades gerais como atenção à saúde, tomada de decisões, comunicação, liderança, administração e educação permanente ${ }^{6}$. De formadiferentede disciplinas prioritariamente clínicas, a saúde col etiva pede uma reflexão especial por tratar de conteúdos inseridos em disciplinas que são desenvolvidas pelaintegração entre profissão e sociedade, num cotidiano compartilhar de saberes e práticas? ${ }^{7}$. Assim, o docente deverátrabaIhar na perspectiva da formação do cidadão, contextualizando técnicas, conhecimentos ehabilidades, considerando o acadêmico com éticaesensibilidade ${ }^{5}$. Busca-se nesteestudo conhecer a percepção e utilização dos conteúdos referentes à saúde col etiva na prática cotidiana de trabal ho do cirurgião-dentista egresso de uma faculdade pública.

\section{Métodos}

0 estudo realizado foi do tipo quanti-qualitativo. A pesquisa qualitativa, segundo Minayo ${ }^{8}$, preocupa-se com a realidade que não pode ser quantificada e aprofunda-se no mundo do significado das relações humanas. Após revisão da literatura, as estratégias utilizadas foram pesquisa, análise documental das Diretrizes Curriculares Nacionais (DCN), Diretrizes Curriculares Nacionais de Graduação em Odontologia (DCNO), planos de ensino, ementas e diários de classe das disciplinas curriculares da área de saúde coletiva e aplicação de questionário.

Foram enviados questionários, com questões abertas efechadas, via e mail, uma única vez aos cirurgiões-dentistas egressos da Faculdade de Odontologia da UFG nos anos de 2000 a 2002. A análise dos dados coletados foi feita por meio da triangulação de técnicas. Para Deslandes ${ }^{9}$, a busca pela triangulação se faz mister especialmente na saúde pública, cujo campo extrapola as fronteiras do biológico, da doença. 0 estudo obtevea aprovação do Comitê de Ética em Pesquisa da Universidade Federal de Goiás. Fizeram parte deste estudo 72 profissionais ea pesquisa foi realizadade junho de 2006 a março de 2007.

\section{Objetivo geral}

Conhecer a percepção eutilização dos conteúdos de saúdecoletiva na prática profissional dos egressos do curso de odontologia da Faculdade de 
Odontologia da FO/UFG, graduados em 2000 2001 e2002.

\section{Objetivos específicos}

Identificar se o egresso está exercendo a profissão e sua adaptação a ela; conhecer a utilização dos conteúdos em saúde coletiva pelos egressos que exercem sua profissão em diferentes locais; verificar se a pós-graduação influencia na utilização dos conteúdos de saúde coletiva; identificar os conteúdos relativos à saúde col etiva recordados e sua utilização pelos egressos; identificar o desenvolvimento de práticas educativas na rotina de trabalho destes profissionais; relacionar os principais fatores percebidos pelos egressos como dificultadores para o desenvolvimento de ações relativas à saúde coletiva; conhecer a percepção sobre a importância dos conteúdos de saúde coletiva perante a atuação no mercado de trabalho; identificar a utilização dos conteúdos de saúde coletiva na prática profissional; conhecer a percepção dos egressos em relação aos estágios curriculares, propostos pelas disciplinas da área de odontologia em saúde coletiva; relacionar os fatores percebidos como facilitadores para o desenvolvimento de práticas em saúde coletiva na rotina profissional; apresentar sugestões que colaborem na melhoria e otimização das disciplinas relacionadas à saúde coletiva, subsidiando a reforma curricular.

\section{Resultadosediscussão}

Observa-se, dentre os participantes, quea maioria $(83,3 \%)$ está exercendo a profissão como cirurgião-dentista. Um número também significativo $(16,7 \%)$ relata não estar mais em exercício profissional da odontologia, o quechama a atenção, uma vez que os egressos pesquisados graduaram-seentreos anos de 2000 e 2002 e a coleta dos dados foi realizada em 2006 (Tabela 1).

Bastos et al. ${ }^{10}$ afirmam que a odontologia passa por uma faseem que os profissionais, principalmente os recém-formados, têm grande dificuldade para se estabelecer no mercado de trabalho, o que desestimula, precocemente, o exercício desta profissão.

Dentre os pesquisados $70,7 \%$ atuam na capital, em clínicas privadas (44,4\% exclusivamente). Os dados encontrados neste estudo referentes à localização dos profissionais nas cidades do interior ou em capitais reforçam estudos semelhantes
Tabela 1. M otivos para não exercer a profissão como cirurgião-dentista. Goiânia (GO), 2006.

\begin{tabular}{lrr}
\hline \multicolumn{1}{c}{ M otivo por não exercer a odontologia } & \multicolumn{1}{c}{$\mathrm{n}$} & $\%$ \\
\hline Trabalha em outra área & 3 & 30,0 \\
Não se adaptou ou não gosta da profissão & 3 & 30,0 \\
Faltam boas condições de trabalho & 1 & 10,0 \\
Está fazendo pós-graduação & 2 & 20,0 \\
Está no exterior & 1 & 10,0 \\
Total & 10 & 100,0 \\
\hline
\end{tabular}

encontrados na literatura consultada, na qual há uma maioria de cirurgiões-dentistas concentrada nas capitais em relação a cidades interioranas ${ }^{11-13}$.

De acordo também com estudos desenvolvidos no campo dos recursos humanos para a saúde no M ercosul, Campos et al. ${ }^{14}$ apontam para a concentração profissional nas capitais e grandes centros urbanos dos países (Paraguai, Uruguai, Argentina eBrasil).

Número significativo destes egressos continuou os estudos cursando pós-graduações, com destaque para as especializações (68,1\%), sendo que ortodontia foi citada como a especialização mais cursada (38,5\%).

Quanto ao desenvolvimento de práticas em saúde col etiva, em suas atividades cotidianas, foi observado que o número daqueles que responderam "sim" (52,2\%) ficou muito próximo dos que relataram "não" (47,8\%). A atuação no serviço público determinou realizações demais práticas caracterizadas como saúde coletiva pelos profissionais.

Os egressos apontaram que têm dúvidas sobre o que sejam as ações em saúde coletiva, embora afirmem que as praticam, indicando que ainda não são claras as terminologias das ações associadas à saúde coletiva entre os pesquisados.

Buscando conhecer os conteúdos que os pesquisados recordavam terem sido ministrados pelas disciplinas da área de saúde coletiva (Educação em Odontologia, O dontologia Social I ell) durante sua graduação, foi elaborada, baseada nos planos deensino das referidas disciplinas, uma relação de temas e, a partir destes, os pesquisados, à medida que recordavam de cada item como ministrado, faziam sua seleção (Gráfico 1 ).

Observou-se que "promoção da saúde" foi recordado por $100 \%$ dos egressos, mas que, destes, apenas $68,7 \%$ relataram utilizar. Esteresultado pôde sugerir que a recordação pode não sig- 


\section{Conteúdos de saúde coletiva}

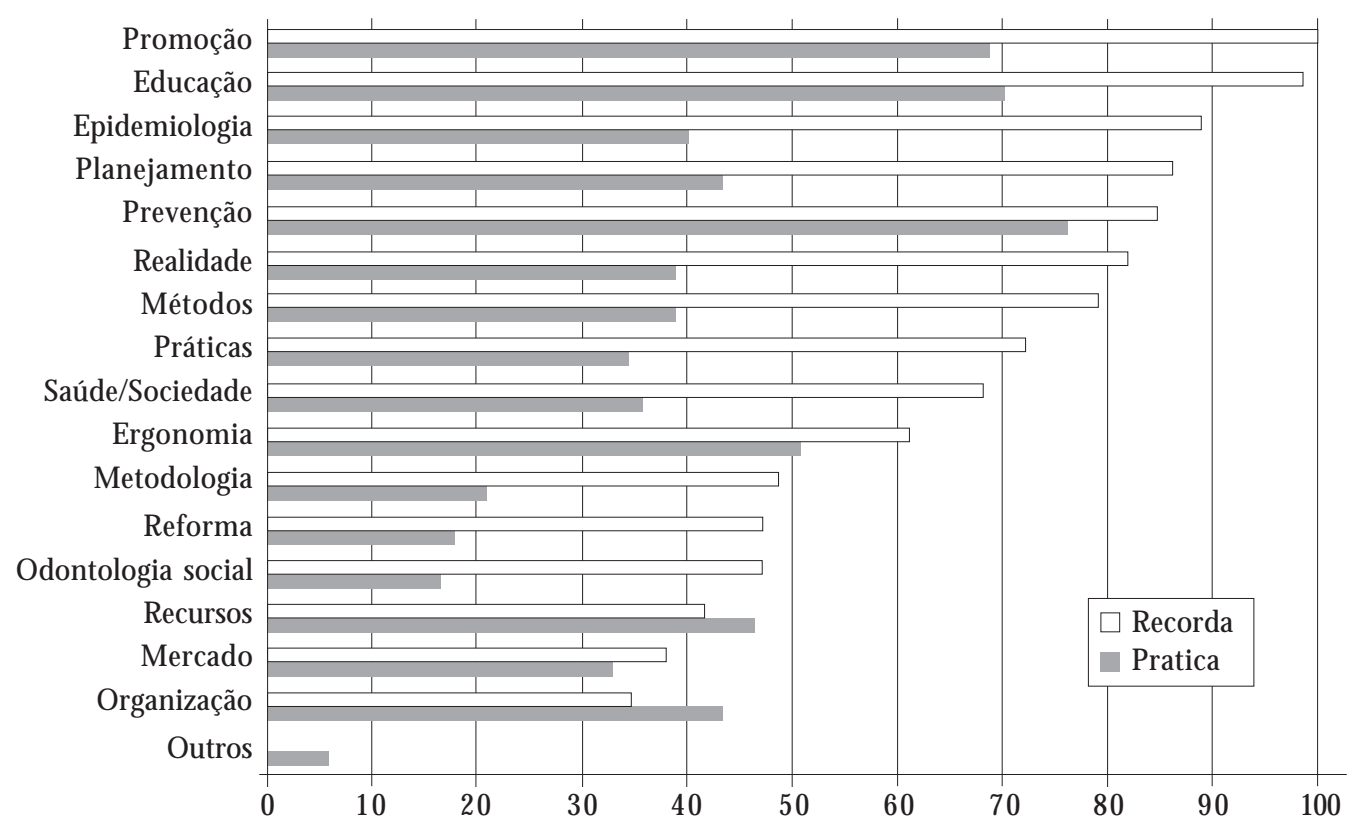

Gráfico 1. Conteúdos de saúde coletiva recordados e utilizados pelos egressos do curso de odontologia. Goiânia (GO), 2006.

nificar apreensão do conteúdo ou ainda que o fato de recordar pode não influenciar na utilização do mesmo.

Dentre as práticas citadas como desenvolvidas pelos participantes, as "palestras" e"orientação/ técnicas de higiene bucal" lideraram as citações com mais de $20 \%$ cada uma delas, totalizando $51,2 \%$.

Tais resultados vêm reforçar que as práticas educativas desenvolvidas predominantemente são verticalizadas, nas quais o profissional, muitas vezes, transmite as informações ao público participante sem, contudo, oportunizar que se estabeleçam as conexões entre conteúdo e contexto.

o campo científico da educação em saúde e da educação popular tem produzido evidências das limitações das práticas educativas centradas na lógica do saber técnico, de natureza normativa eque não consideram o cotidiano emodos de vida de indivíduos e coleti vidades ${ }^{15}$.

Outras possibilidades de construção do conhecimento em saúde de forma mais participativa foram propostas pelo gestor federal do SUS, na Política de Gestão Estratégica e Participativa no SUS ${ }^{16}$.
Sobre os fatores que representam principais entraves para o desenvolvimento de ações relativas à saúde coletiva em seu cotidiano profissional, os mais citados foram a falta de recursos materiais complementares, dificuldade de valorização das atividades em saúde coletiva, a falta de tempo para realização destas atividades e infraestrutura precária para a realização das atividades em saúde coletiva.

A forte influência da tecnologia dura na prática odontológica, reforçada dentro das faculdades de odontologia, através dos currículos e das atividades clínicas de alta sofisticação, como tem sido até então, pode explicar a dificuldade de superação destes entraves.

Os currículos da área de odontologia, organizados privilegiando a doença em vez da saúde, criam a necessidadee dependência de uso da tecnologia dura, pois de forma geral esta atua nos estágios em que a doença já está instalada ou mesmo em fases avançadas.

Um currículo deve comprometer-se com a democratização efetiva da sociedade, formando profissionais que visualizem sua posição na estrutura social e com condições clara de optarem 
por uma cidadania respon sável e participativa ${ }^{17,18}$ Estudos desenvolvidos por alguns autores podem corroborar com essa idéia de que as ações odontológicas simplesmentese extinguem quando não há equipamento, material ou instrumental. Isso traz como consequência a perda da dimensão de uma profissão de saúde, que deveria priorizar a atenção integral, começando pelos aspectos de prevenção e promoção da saúde, que são pouco dependentes de tecnologia avançada ou complexa ${ }^{19,20}$.

Como fatores facilitadores de práticas em saúde coletiva na rotina profissional, identificaram-se oito aspectos, agrupados em dois grandes grupos, um relativo ao próprio profissional (conscientização dos profissionais e remuneração) e outro relativo ao desempenho profissional, incluindo os aspectos das políticas públicas (educação, promoção, insumos e financiamento) e da forma de gerenciamento (individualização do atendimento e condições de trabalho).

No que diz respeito ao próprio profissional, o primeiro aspecto é a conscientização destes. Ficou evidente que, para os entrevistados, não há uma clareza sobre a importância e a necessidade de que sejam desenvolvidas práticas que configuram a saúde coletiva dentro da rotina profissional. Isso pode ser, pela formação, como têm sido construídos e trabalhados os currículos nas instituições deensino superior, com ênfase no biológico, em que cada disciplina se apre senta durante 0 curso de forma pontual, sem inter-relações entreas mesmas ou com o contexto. Ainda, também pode ser sugestivo de que 0 conceito acerca do que seja saúde coletiva não esteja claro entre os egressos.

"A expressão saúde coletiva é uma invenção tipicamente brasileira que surgiu em fins da dé cada de 1970, na perspectiva de constituir um paradigma que permitisse uma nova articulação entre as diferentes instituições do campo da saúde"$^{\prime 21}$. Para a autora, a saúde coletiva abrange atualmente um conjunto complexo de saberes e práticas relacionados ao campo da saúde, envolvendo desde organizações que prestam assistência à saúde da população atéinstituições de ensino e pesquisa e organizações da sociedade civil.

Ressalta-se outro importante aspecto mencionado entre os participantes, que foi a forma de gerenciamento (individualização do atendimento e condições de trabalho). E, sobre isso, considerando as transformações ocorridas no mundo do trabalho, as DCN propõem como competências gerais para os profissionais de odontologia que estes estejam aptos a realizar o gerencia- mento eadministração da força de trabal ho, dos recursos físicos e materiais e da informação, além de ser gestores, empregadores ou lideranças na equipe de saúde ${ }^{6}$.

A formação dos profissionais de saúde tem permanecido alheia à organização da gestão setorial e ao debate crítico sobre os sistemas de estruturação do cuidado ${ }^{22}$.

A saúde exige cada vez mais do profissional uma postura ética e de responsabilidade, reconhecendo no usuário dos serviços de saúde alguém repleto de necessidades complexas, cuja necessidade principal às vezes pode não ser a doença. N esta perspectiva, a equipedesaúdenão deve concentrar suas atividades apenas em procedimentos técnicos, mas também procurar refletir e atuar considerando a importância do envolvimento com o usuário.

Cada vez são mais tênues os limites entre disciplinas, entreas ciências sociais, biológicas eexatas e cresce de importância o desafio de a ciência ser capaz de dialogar com todas as formas de conhecimento, no sentido de ampliar sua capacidade de explicação ${ }^{23}$.

Deacordo com os conteúdos de saúde coletiva ministrados durante o curso de graduação, relacionando-os com a atuação do profissional no mercado de trabalho, 97,2\% reconhecem a importância dos mesmos, sendo que, para $52,1 \%$, esses conteúdos são "muito importantes" e, para 45,1\%, considerados "importantes" (Gráfico 2).

Tabela 2. Fatores que representam dificultadores para ações de saúde coletiva. Goiânia (GO), 2006.

\begin{tabular}{lccc}
\hline \multicolumn{1}{c}{ Fator } & $\mathrm{n}$ & $\%$ & Total \\
\hline Falta de recursos materiais complementares & 28 & 41,8 & 67 \\
Dificuldade de valorização desta ação pela & 27 & 40,3 & 67 \\
população & & & \\
Falta de tempo & 26 & 38,8 & 67 \\
Infraestrutura precária das unidades de saúde & 25 & 37,3 & 67 \\
Condições de trabalho precárias & 15 & 22,4 & 67 \\
Não se sentir preparado & 11 & 16,4 & 67 \\
Vínculo empregatício & 11 & 16,4 & 67 \\
Falta de conhecimentos técnicos & 4 & 6,0 & 67 \\
Não ter contato com pacientes da saúde & 2 & 3,0 & 67 \\
pública & & & \\
Dificuldade para falar em público & 2 & 3,0 & 67 \\
Desmotivação & 2 & 3,0 & 67 \\
Falta supervisão & 1 & 1,5 & 67 \\
Burocracia & 1 & 1,5 & 67 \\
\hline
\end{tabular}




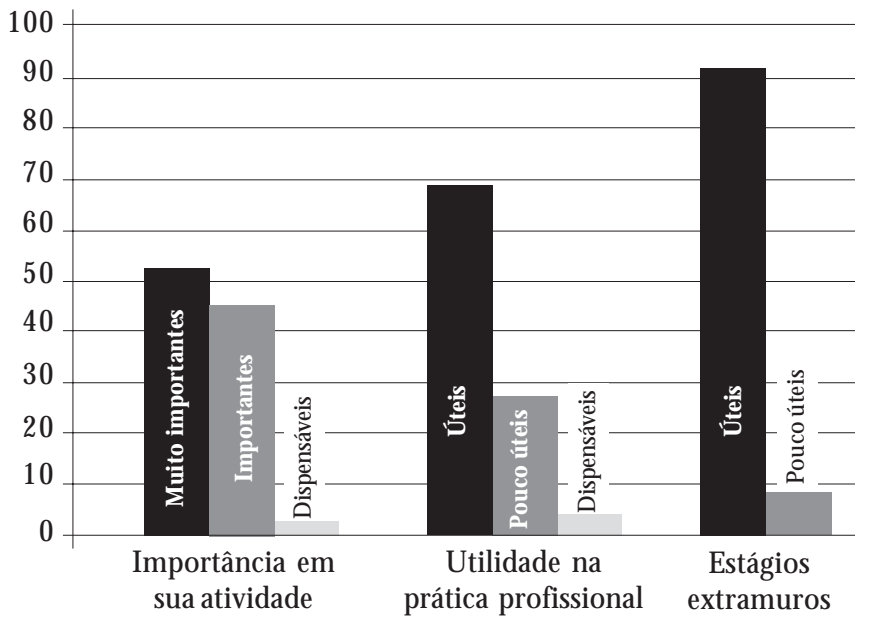

Gráfico 2. Comparação entre a importância dos conteúdos, utilização e estágio extramuros em saúde coletiva. Goiânia (GO), 2006. proporcionar na preparação do futuro cirurgiãodentista para seu campo de atuação.

Foi solicitado dos participantes que apresentassem sugestões de conteúdos que pudessem colaborar para a melhoria e otimização das disciplinas relacionadas à saúde coletiva. As sugestões apresentadas foram de conteúdos teóricos e também de estratégias.

Dentreas sugestões de conteúdos, emergiram oito grandes grupos que dizem respeito a mercado de trabalho, ergonomia, administração de consultórios, humanização nos serviços odontológicos, gestão de serviços, marketing, promoção da saúde em consultório privado, recursos humanos. Confrontando estas sugestões com os planos decurso eementas das disciplinas deEducação em O dontologia, O dontologia Social I ell, e objetos da análise documental, foi observado que muitos destes conteúdos foram ministrados durante a graduação, porém, dado às sugestões apresentadas, parece que os profissionais não se sentiram suficientemente contemplados.

\section{Consideraçõesfinais} pôde considerar que a proposta de atuação nas atividades práticas (estágio curricular extramuros) desenvolvidas na Faculdade de O dontologia da UFG, pelas disciplinas de saúde coletiva, foram bastante válidas para os participantes.

Os espaços de realização dos estágios curriculares supervisionados têm constituído num lugar privilegiado para reflexão acerca de possíveis transformações na forma de se oferecer saúde, na medida em que, em contato com a realidade, os professores, os alunos e os profissionais de saúde dos serviços locais podem ser despertados para a necessidade de (re) pensar, de forma integrada e contextualizada, a melhor forma de promover a saúde no nível local.

As atividades práticas extramuros dentro dos cursos de graduação na área da saúde podem colaborar proporcionando a aquisição deconhecimentos, habilidades e atitudes na vivência de um mundo real, onde a integração da universidade com a comunidade é muito importante e necessária, pois o futuro profissional, em contato com comunidades em seus contextos, além do aprendizado, pode também praticar o exercício da cidadania, possibilitando a construção de um profissional mais humano.

0 contato do aluno de graduação junto à realidade dos serviços, saindo dos espaços da universidade, apresenta-se como uma importante atividade que o curso de odontologia pode
Com base nos dados coletados, pôde-se delinear o perfil do egresso deste estudo concluindo quea maioria (83,3\%) está atuando como cirurgiãodentista; destes, 70,7\% atuam na capital, em clínicas privadas $(44,4 \%)$; um número significativo destes egressos continuou os estudos cursando pós-graduações, com especialização liderando $(68,1 \%)$. A ortodontia foi citada como a especialização mais cursada $(38,5 \%)$ entre os profissionais pesquisados.

Pôde-se concluir que o egresso tem dúvidas sobre o que sejam as ações em saúde coletiva, embora as pratique. A pesquisa indica que ainda não são claras as terminologias das ações associadas à saúde coletiva em relação ao exercício destas entre os pesquisados.

0 fato de o profissional atuar no serviço público determinou realizações de mais práticas caracterizadas como saúde coletiva, foi significativa a realização entre profissional de clínica pública em relação ao de clínica privada.

Dentre os fatores citados como dificultadores ao desenvolvimento de ações em saúde coletiva, a falta de recursos materiais complementares, a dificuldade devalorização destas ações pela população, a falta de tempo e a infraestrutura precária das unidades de saúde representaram os mais citados empecilhos a não real izações destas ações no cotidiano profissional. 
Foi possível identificar os conteúdos ministrados durante a graduação; porém, vários destes, embora registrados nos planos de curso e diários de classe como ministrados, não foram associados pelo egresso como relativos à saúde coletiva. Dentre os conteúdos recordados pelos pesquisados como associados à saúde coletiva, "promoção da saúde" foi recordado por todos.

Ainda educação em saúde, epidemiologia, planejamento, prevenção e realidade de saúde bucal no Brasil foram conteúdos recordados por mais de $80 \%$ dos participantes.

Com relação aos conteúdos utilizados, prevenção em saúde foi o mais citado, sendo ainda educação em saúde, promoção e ergonomia os conteúdos que são relatados como sendo utilizados nas práticas cotidianas por mais de $60 \%$ dos egressos.

Sobreos estágios curriculares, o estudo apontou uma enorme valorização desta atividade entre os pesquisados; $91,7 \%$ destes acham válidas as práticas extramuros. Registrou-se também 0 desejo entre os participantes de terem aproveitado mais as práticas extramuros.

Conclui-se, então, já ser consenso entre os egressos a importância de práticas em saúdejunto às comunidades durante a formação.

Todos os profissionais que fazem parte do Programa de Saúde da Família relataram práti- cas cotidianas em saúde coletiva. Conclui-se então haver diferença de realização de algum tipo de prática em saúde coletiva entre os profissionais nas esferas pública e privada.

Analisando-se a cidade de atuação - capital ou interior - e pós-graduação, pôde-se concluir que não houve diferença quanto à utilização dos conteúdos entre esses profissionais.

As sugestões de conteúdos e estratégias apresentadas, apesar demuitas destas, de acordo com planos de ensino e documentos analisados, já terem sido ministradas durante a graduação, poderão representar valiosa colaboração para otimizar as disciplinas de saúde coletiva, na medida em que podem indicar necessidade de que seja revisto o enfoquee possíveisnecessidades demaior contextualização destes conteúdos no momento de reforma curricular nos diversos cursos.

Desde o final da década de noventa, a Faculdade de O dontologia da UFG oferece cursos de pós-graduação lato sensu em odontologia em saúde coletiva e, atualmente, tem linha de pesquisa e orientação de mestrado na área.

Este estudo sugere a investigação das contribuições das atividades de extensão e pesquisa na construção dos conteúdos de saúde coletiva na graduação, principalmenteno quese refereàquelas de natureza interdisciplinar.

\section{Colaboradores}

DE Badan trabalhou na execução da pesquisa, elaboração e redação final do texto. VC M arcelo orientou todas as etapas da pesquisa e da elaboração e redação final do texto. DG Rocha participou da revisão bibliográfica, organização e redação final do texto. 


\section{Referências}

1. Sheiham A, Moysés SJ. O papel dos profissionais de Saúde Bucal na Promoção de Saúde. In: Buischi YP. Promoção de Saúde Bucal na Clínica O dontológica. São Paulo: Artes M édicas/EAP-APCD; 2000. p. 26-36.

2. Freire MCM. Dieta, Saúde Bucal e Saúde Geral. In: Buischi YP. Promoção de Saúde Bucal na Clínica O dontológica. São Paulo: Artes M édicas/EAP-APCD; 2000. p. 275.

3. Teixeira M CB. A dimensão cuidadora do trabalho de equipe em saúde e sua contribuição para a odontologia. Cien Saude Colet [periódico na Internet] 2006 [acessado $2006 \mathrm{dez}$ 17];11(1):[cerca de 7 p.]. Disponível em: http://www.scielo.br/pdf/csc/v1ln1/ 29447.pdf

4. Paim JS. Collective health and the challenges of practice In: Pan American Health Organization. The crisis of public health: reflections for the debate. Washington, D.C.: Pan American Health Organization; 1992. p. 136-50. [Scientific Publication 540]

5. Narvai PC, Frazão P. Epidemiologia, política, e saúde bucal coletiva. In: Antunes JLF, Peres MA. Epidemiologia da saúde bucal. Rio de Janeiro: Guanabara Koogan; 2006.

6. Brasil. Conselho Nacional de Educação. Câmara de Educação Superior. Resolução № CNE/CES 3, de 19 de fevereiro de 2002. Institui Diretrizes Curriculares N acionais do Curso de Graduação em Odontologia. Diário Oficial da União 2002; 04 mar.

7. Coêlho IM. Graduação: rumos e perspectivas. Revista da rede de avaliação institucional da educação superior 1998; 3(3):80.

8. Minayo MCS. Triangulação de M étodos em Avaliação de Programas e Serviços de Saúde. In: IV Congresso Latinoamericano de Ciencias Sociales y M edicina; 1997; Cocoyoc, M éxico.

9. Deslandes SF, Assis SG. Abordagens quantitativa e qualitativa em saúde: 0 diálogo das diferenças. In: M inayo MCS, Deslandes SF, organizadoras. Caminhos do pensamento: epistemologia e método. Rio de Janeiro: Fiocruz ; 2002. p. 195-223.

10. Bastos JRM, Aquilante AG, Lauris JRP, Almeida BS, Bijela VT. Análise do perfil profissional de cirurgiões-dentistas graduados na Faculdade de Odontologia de Bauru- USP entre os anos de 1996 e 2000. J. Appl Oral Sci [periódico na Internet] 2003 [acessado 2006 set 05];11(4):[cerca de7 p.]. Disponível em: http://www.scielo.br/pdf/jaos/v1ln4/a02v1ln4.pdf

11. Cordón J. A saúde bucal e mercado de trabalho odontológico. Saúde em Debate [periódico na Internet] 1986 [acessado 2006 out 24];18:[cerca de 13 p.]. Disponível em: http://www.scielo.br/pdf/csc/ v9n1/19828.pdf
12. Costa B, Stegun RC, Todescan R. Do ensino à prática odontológica: um levantamento da realidade na grande São Paulo. Rev. Assoc. Paul. Cirurg. Dent. 1993; 46(6):909-913.

13. Narvai PC. Recursos humanos para a promoção da saúde bucal. In: Krieger L, organizador. Promoção de Saúde Bucal. São Paulo: Artes M édicas; 1997. p. 447-463.

14. Campos F, Brito P, Rígoli F. 0 campo dos recursos humanos para a saúde no M ercosul. [acessado 2006 out 24]. Disponível em: http://www.opas.org.br/ $\mathrm{rh} /$ publicacoes/textos_apoio/rhm_campo.pdf

15. Brasil. Ministério da Saúde. Secretaria de Gestão Estratégica e Participativa. Departamento de Apoio à Gestão Participativa. Caderno de educação popular e saúde. Brasília: Ministério da Saúde; 2007.

16. Brasil. M inistério da Saúde. Secretaria de Gestão Estratégica e Participativa. ParticipaSU S. Política N acional de Gestão Estratégica e Participativa no SU S. Brasília: M inistério da Saúde; 2007.

17. Freire P. Educação e mudança. 17 a ed. Rio de Janeiro: Paz e Terra; 1979.

18. Brasil. M inistério da Saúde. Política Nacional de Educação Permanente em Saúde. Portaria N o 198/ GM/MS. Diário Oficial da União 2004. 13 fev.

19. Unfer B, Saliba O. O cirurgião-dentista do serviço público: formação, pensamento e ação. Rev Bras O dont Saúde Coletiva 2001; 2(2):45-51.

20. Rocha DG, Lima JR, M ello L, Batista IV. Diversidade e equidade no SUS: parceria universidade e educação popular. Goiânia: Cânone Editorial; 2008.

21. L'Abbate S. A análise institucional e a saúde coletiva. Cien Saude Colet [periódico na Internet] 2003 [acessado $2006 \mathrm{dez}$ 16];8(1):[cerca de 10 p.]. Disponível em: http://www.scielo.br/pdf/csc/v8n1/ a19v08n1.pdf

22. Ceccim RB, Feuerwerker LCM . O quadrilátero da formação para a área da saúde: ensino, gestão, atenção e controle social. Physis (Rio J.) 2004; 14(1):41-65.

23. Feuerwerker LCM. Educação dos profissionais de Saúde hoje - problemas, desafios, perspectivas e as propostas do M inistério da Saúde. Rev. ABEN O [periódico na Internet] 2003 [acessado 2006 dez 15]; 3(1):[ cerca de 4 p./. Disponível em: http://www. abeno.org.br/revista/arquivospdf/2003/feue.pdf

Artigo apresentado em 24/10/2007

Aprovado em 17/07/2008 